\title{
Family meals and body weight in US adults
}

\author{
Jeffery Sobal* and Karla Hanson \\ Division of Nutritional Sciences, Room 303 MVR Hall, Cornell University, Ithaca, NY 14853, USA
}

Submitted 6 May 2010: Accepted 4 January 2011: First published online 28 February 2011

\begin{abstract}
Objective: Family meals are an important ritual in contemporary societies and many studies have reported associations of family meals with several biopsychosocial outcomes among children and adolescents. However, few representative analyses of family meals have been conducted in samples of adults, and adults may differ from young people in predictors and outcomes of family meal consumption. We examined the prevalence and predictors of adult family meals and body weight outcomes.

Design: The cross-sectional 2009 Cornell National Social Survey (CNSS) included questions about the frequency of family meals, body weight as BMI and sociodemographic characteristics.

Setting: The CNSS telephone survey used random digit dialling to sample individuals. Subjects: We analysed data from 882 adults living with family members in a nationally representative US sample.

Results: Prevalence of family meals among these adults revealed that 53\% reported eating family meals seven or more times per week. Predictive results revealed that adults who more frequently ate family meals were more likely to be married and less likely to be employed full-time, year-round. Outcome results revealed that the overall frequency of family meals among adults was not significantly associated with any measure of body weight. However, interaction term analysis suggested an inverse association between frequency of family meals and BMI for adults with children in the household, and no association among adults without children.

Conclusions: These findings suggest that family meals among adults are commonplace, associated with marital and work roles, and marginally associated with body weight only in households with children.
\end{abstract}

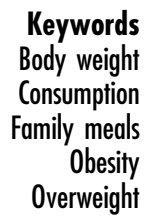

Family meals are an important social activity that may influence dietary intake and health. Many quantitative studies have examined family meals among children and adolescents, but few investigate the prevalence, predictors and outcomes of family meals among adults. The present analysis seeks to begin to fill that knowledge gap by analysing a large, contemporary US survey to examine the frequency of family meals among adults, the characteristics of adults who more or less often eat family meals, and the association between frequency of family meals and body weight.

\section{Prevalence of family meals}

Studies of the prevalence of family meals among children and adolescents report a wide variation of findings, partly because of measurement differences in survey question wording and dissimilarity of the samples. For example, some studies use questions that assess only dinner/supper family meals ${ }^{(1-3)}$, while some consider all meals that could be family meals ${ }^{(4-7)}$. Other investigations examine samples of very different ages. Overall, studies of family meals are more frequent among younger than older children and adolescents, with the majority of US young people eating family dinners together five or more days per week ${ }^{(1,4,8,9)}$.

Studies of adult family meal prevalence in different nations report a prevalence of $46 \%$ to $70 \%$ of families eating a daily main meal together ${ }^{(10-12)}$. In the USA, one study reported that $34 \%$ of adults definitely agreed that 'Our whole family eats together' ${ }^{(13)}$, while another noted that $75 \%$ stated they ate together as a family at least five nights per week ${ }^{(14)}$. Two analyses of family meal consumption by parents of adolescents found that over half of parents and about half of their adolescent children reported they ate family meals on four or five days per week ${ }^{(5,6)}$. Overall, studies of the prevalence of family meal consumption reveal a wide range of findings for samples of children, adolescents and adults in several nations.

\section{Predictors of family meals}

Studies of the predictors of family meals have focused upon sociodemographic variables among children and adolescents. A review of Minnesota studies of family meals in 
adolescents $^{(4)}$ reported that girls, younger adolescents, Asian-Americans, those of higher socio-economic status and those whose mothers were unemployed reported they more frequently ate family meals. However, a review of research about family meals in the USA concluded that few characteristics of children were related to family meal participation ${ }^{(15)}$. These findings about children and adolescents suggest that while gender, age, ethnicity, socio-economic status and work status may be predictors of the frequency of family meals, existing evidence is inconclusive.

Few analyses examine predictors of family meals among adults. In four Nordic nations ${ }^{(12)}$ no gender differences existed in frequency of family meals, but adults who were older, less educated, not employed and in working class occupations more often ate family meals.

The extent to which sociodemographic variations in family meals is consistent or incongruous between adults and adolescents remains unclear. Furthermore, it is not clear how sociodemographic variables like marital status and parenthood that are not relevant to assess in studies of children and adolescents may be associated with frequency of adult family meals.

\section{Body weight outcomes of family meals}

A growing body of research has quantitatively analysed the relationships between frequency of family meals and body weight among children and adolescents. Most of these studies are cross-sectional surveys that have reported an inverse relationship between frequency of family meals and various measures of body weight ${ }^{(1,3,7,16-18)}$, although some studies have reported that the inverse relationship was limited to one gender ${ }^{(7,19,20)}$, ethnic group ${ }^{(21)}$ or social class category $^{(22)}$. In contrast, other cross-sectional studies have reported no association between family meal frequency and measures of body weight ${ }^{(8,23-27)}$ or a direct association in some subgroups ${ }^{(22)}$. A smaller number of longitudinal studies have examined frequency of family meals and change in body weight in children and adolescents over periods that ranged from 1 to 5 years, and have reported inverse associations $^{(1,24,28)}$ or no significant associations ${ }^{(3,7)}$.

Few studies provide quantitative data about how family meals are related to body weight among adults; those that do suggest that the association may be different for adults and young people. For example, a survey of students reported that eating fast food for family meals was not significantly associated with body weight for those adolescents, but more frequent fast food for family meals was related with higher body weight for their parents ${ }^{(29)}$.

\section{The need for research about adult family meals and body weight}

Almost all existing quantitative studies of family meals and body weight sampled only children and adolescents, and sometimes their parents. However, findings drawn only from young people are limited in several ways, including: (i) representativeness of samples focusing on children and adolescents; (ii) divergence between young people and adults in experiencing and reporting about family meals; and (iii) differential effects of family meals on young people and adults.

The representativeness of child and adolescent samples is limited because they include only a portion of all families. The 2000 US Census found that households included $26 \%$ with one individual living alone, $21 \%$ with two spouses with no children, $27 \%$ with two parents with children, 10\% with one parent with children, 3\% with unmarried partners with no children and $13 \%$ other configurations ${ }^{(30)}$. Many families include only adults with no children in the household as childless couples or those whose children have grown and left home. The prevalence, predictors and outcomes of family meals for adults without children may differ from those of households that include children ${ }^{(13)}$.

Divergence in family meal participation, experience and interpretation may occur between young people and adults. Compared with their parents, children and adolescents in the same household report eating fewer family meals, think family meals are less important, and are less sensitive to problems in scheduling family meals ${ }^{(5,6)}$. Adolescents often have educational, extracurricular, employment and social involvements that prevent them from eating family meals, even when the parents and other family members eat together.

Differential effects of family meals may occur when joint meals provide benefits to young people that may not be beneficial and may even be disadvantageous for adults. Understanding family meals among many age categories, including adults, is important because family meals involve all members of the family. A suggestive example of such differential effects was seen in a study that found no association of frequency of family meals with adolescents' weights, but higher weights among parents eating some types of family meals ${ }^{(29)}$.

These and other limitations in existing research suggest that new studies of adult family meals are essential to more fully understand family meal prevalence, predictors and outcomes. Adult research is needed to complement and extend the substantial body of research in samples of children and adolescents.

We hypothesized that the prevalence of family meals among adults would be high, with most eating with other family members daily. We hypothesized that predictors of adult family meals would be congruent with Holme's ${ }^{(12)}$ finding of more frequent family meals among older, less educated and unemployed adults, with no gender differences. We further hypothesized that whites more often eat family meals compared with black and Hispanic adults, who marry less frequently ${ }^{(31)}$. We anticipated that married people more often eat family meals due to expectations about joint eating ${ }^{(32)}$ and that parents more often eat family meals based on child-rearing norms that 
prescribe joint eating ${ }^{(33)}$. We hypothesized that, as with adolescents, the more often adults eat family meals the lower will be their body weights, and the less often they eat family meals the more likely that they will be overweight or obese.

\section{Methods}

\section{Design and sample}

Data for the present analysis were drawn from the Cornell National Social Survey (CNSS), an annual pilot-tested cross-sectional telephone survey conducted by the Survey Research Institute (SRI; Ithaca, NY, USA). The CNSS sample was collected in November and December of 2009 using random digit dialling to select households with listed and unlisted telephone numbers in the Continental USA provided by GENESYS Sampling Systems (Fort Washington, PA, USA). Within contacted households, the adult with the most recent birthday was sampled. Telephone interviews were conducted in English by trained interviewers using computer-assisted telephone interviewing software. The final sample size for the 2009 CNSS was 1000 completed interviews, with a cooperation rate (completed interviews/potential interviews) of $61 \%{ }^{(34)}$. The study was conducted in accordance with the Cornell University Institutional Review Board procedures about informed consent and data confidentiality.

\section{Measures}

Frequency of family meals was assessed using an item adapted for adults from the widely used question in the University of Minnesota EAT (Eating Among Teens) studies $^{(4)}$. The CNSS question asked: 'In a typical week, how often do you eat a meal together with the family members who currently live with you? Never, 1 or 2 times, 3 or 4 times, 5 or 6 times, 7 times, over 7 times'. The inclusion of the 'over 7 times per week' category permitted family meals to be reported beyond dinner/supper meals. In addition to these six frequency categories, respondents also could reply that the question was not applicable because they were not living with family. The midpoint of each class interval (with $>7$ set to 9 meals) was used to construct a continuous measure of frequency of family meals per week.

Sociodemographic predictors were assessed using direct questions about the respondents' gender, age in years, ethnicity (white, black, Hispanic, other/multiple), marital status (married or not), presence of children under 18 years of age in the household (present or not), years of educational attainment and employment (employed fulltime year-round or not). These dichotomous measures were used because of limited sample sizes available for finer categorization. Also, the CNSS data analysed here did not differentiate household categories in families without children present, i.e. those who were childless non-parents $v$. those who were parents whose children had grown and left home.

Body weight outcomes were assessed with three measures. Questions asked respondents to self-report their height and weight, which were used to construct a continuous measure of BMI (as $\left.\mathrm{kg} / \mathrm{m}^{2}\right)^{(35)}$. Self-reported height and weight may include reporting bias ${ }^{(36)}$, although one study reported high correlations between measured and self-reported weight $(r=0.97)$ and height $(r=0.93)^{(37)}$. In the present analysis, under-reported weight would lead to underestimated associations between family meals and body weight. Dichotomous indicators of overweight $\left(\mathrm{BMI} \geq 25 \mathrm{~kg} / \mathrm{m}^{2}\right)$ and obesity $\left(\mathrm{BMI} \geq 30 \mathrm{~kg} / \mathrm{m}^{2}\right.$ ) were constructed from BMI ${ }^{(35)}$ to examine both slightly heavier overweight individuals as well as much heavier obese individuals. Our sample had a slightly higher prevalence of overweight and obesity than national US data ${ }^{(38)}$.

\section{Analysis}

Missing values led to exclusion of thirty-four cases, resulting in an initial analytic sample of 966. An additional eighty-two cases reported having no family with whom to eat a family meal, and we excluded these cases from predictor and outcome analyses. We report significant main effects at $P<0.05$ or less and interactions at $P<0 \cdot 10^{(39)}$.

Descriptive analyses examined the frequency of all variables to characterize the sample and provide the overall prevalence of the frequency of family meals. We compared respondents who reported they were not living with family members with whom they could eat family meals with all others, and differences were tested using $\chi^{2}$ analysis and $t$ tests.

Predictor analyses examined the association between frequency of family meals and seven sociodemographic characteristics in the CNSS data using multiple linear regression analysis.

Outcome analyses examined whether the frequency of family meals was associated with three measures of body weight. Multiple linear regression was used to model the frequency of family meals as a predictor of BMI adjusting for all sociodemographic variables; and separate multiple logistic regressions were used to model the frequency of family meals as a predictor of overweight and obesity adjusting for all sociodemographic variables. In addition to the main effects of each sociodemographic variable, a two-way interaction term between having children in the household and family meals also was included in the outcome models in order to test potential differences in the relationship between family meals and body weight in adults with and without children. These interaction analyses will help to consider the extent to which prior studies of family meals among children and adolescents can be generalized to families that have no children at home. 


\section{Results}

\section{Respondents without family members}

Almost 9\% of this sample reported that they had no family members living in their household with whom to eat family meals. Compared with respondents with family, those without family with whom to share meals were significantly older and less likely to be married, to live with children or to be employed full-time year-round (Table 1).

\section{Sample characteristics}

In the remaining analytic sample (Table 1), slightly over half were women, the mean age was about 50 years, over threequarters were white, most were married, under half had children in the household, on average they had education past secondary school, almost half were employed full-time year-round, most were overweight and a fifth were obese.

\section{Prevalence of family meals}

On average, respondents ate six family meals per week. The overall prevalence of family meals included $7 \%$ of respondents reporting they never ate family meals, $21 \%$ stating they ate family meals seven times per week, and $32 \%$ more than seven times weekly (Table 1 ).

\section{Predictors of family meals}

Being married was a strong, direct predictor of frequency of family meals, with married respondents eating about two more family meals per week than unmarried respondents $(+1 \cdot 99, P<0 \cdot 01$; Table 2$)$. Full-time, yearround employment was a significant inverse predictor of family meals $(-0 \cdot 65, P<0 \cdot 01)$. Other sociodemographic variables were not significantly associated with family meal frequency.

\section{Outcomes of family meals}

The frequency of family meals was not significantly associated with BMI, overweight or obesity in multivariate linear and logistic regression analysis adjusting for sociodemographic variables (Table 3). However, the interaction term between family meals and children in the household was associated with BMI $(P<0 \cdot 10)$. In this model, adults in households with children had marginally higher BMI than adults in childless households $(+1 \cdot 72$, $P<0 \cdot 10$ ), but more frequent family meals in this group were associated with lower BMI compared with households without children, where family meals were not associated with BMI (Fig. 1).

\section{Discussion}

The present study is unique as one of the first analyses that focus on family meals and body weight among a large representative sample of US adults, when most prior studies of family meals have focused on children and adolescents. While there were some similarities between these findings about adult family meals and studies of adolescents, several differences also existed.

Table 1 Characteristics of respondents, body weight and prevalence of family meals, by having someone with whom to eat family meals: nationally representative US adult sample, 2009 Cornell National Social Survey

\begin{tabular}{|c|c|c|c|}
\hline & $\begin{array}{l}\text { No one with whom to } \\
\text { eat family meals } \\
(n \text { 84) }\end{array}$ & $\begin{array}{l}\text { Someone with whom to } \\
\text { eat family meals } \\
(n \text { 882) }\end{array}$ & Significancet \\
\hline \multicolumn{4}{|l|}{ Characteristic } \\
\hline Female (\%) & $52 \cdot 4$ & $52 \cdot 9$ & \\
\hline Mean age (years) & $62 \cdot 7$ & $49 \cdot 2$ & ** \\
\hline \multicolumn{4}{|l|}{ Race/ethnicity (\%) } \\
\hline White, non-Hispanic & $85 \cdot 7$ & $78 \cdot 7$ & \\
\hline Black, non-Hispanic & $7 \cdot 1$ & $7 \cdot 1$ & \\
\hline Hispanic & $2 \cdot 4$ & $7 \cdot 4$ & \\
\hline Other/multiple & $4 \cdot 8$ & $6 \cdot 8$ & \\
\hline Married (\%) & $2 \cdot 4$ & $69 \cdot 3$ & ** \\
\hline Any children in household (\%) & $0 \cdot 0$ & $43 \cdot 1$ & ** \\
\hline Mean years of education & $14 \cdot 4$ & $14 \cdot 6$ & \\
\hline Employed full-time, year-round (\%) & $31 \cdot 0$ & $46 \cdot 7$ & ** \\
\hline \multicolumn{4}{|l|}{ Body weight‡ } \\
\hline Mean BMI $\left(\mathrm{kg} / \mathrm{m}^{2}\right)$ & $27 \cdot 1$ & $26 \cdot 6$ & \\
\hline Overweight (\%) & $58 \cdot 8$ & $57 \cdot 1$ & \\
\hline Obese (\%) & $26 \cdot 3$ & $20 \cdot 4$ & \\
\hline \multicolumn{4}{|l|}{ Family meals per week (\%) } \\
\hline 0 & - & $6 \cdot 7$ & \\
\hline 1 or 2 & - & $7 \cdot 9$ & \\
\hline 3 or 4 & - & $13 \cdot 8$ & \\
\hline 5 or 6 & - & $18 \cdot 9$ & \\
\hline 7 & - & $20 \cdot 7$ & \\
\hline$>7$ & - & 31.9 & \\
\hline
\end{tabular}

Significance: ${ }^{*} P<0.05,{ }^{* *} P<0.01$.

tDifferences were tested using $t$ tests and $\chi^{2}$ analysis.

‡BMI data are available for 900 respondents. 
Table 2 Multivariate model of demographic predictors of frequency of family meals: nationally representative US adult sample, 2009 Cornell National Social Survey

\begin{tabular}{lcc}
\hline & Frequency of family meals & Significancet \\
\hline Constant & $(n$ 882) & $* *$ \\
Female & 4.90 & \\
Age & -0.26 & \\
Black, non-Hispanic & -0.00 & $*$ \\
Hispanic & 0.37 & $*$ \\
Other/multiple race & -0.19 & \\
Married & -0.06 & \\
Any children in household & 1.99 & $* *$ \\
Years of education & 0.41 & $* .00$ \\
Employed full-time, year-round & -0.65 & \\
\hline
\end{tabular}

Significance: ${ }^{\star} P<0.05,{ }^{\star \star} P<0.01$.

†The statistical significance of regression coefficients was examined using $t$ tests.

$¥$ White, non-Hispanic is the reference group.

Table 3 Multivariate model of body weight by frequency of family meals and demographic characteristics, and the interaction between having any children at home and frequency of family meals: nationally representative US adult sample, 2009 Cornell National Social Survey

\begin{tabular}{|c|c|c|c|c|c|c|}
\hline & $\begin{array}{c}\mathrm{BMI} \\
(n \text { 820) }\end{array}$ & Significancet & $\begin{array}{l}\text { Overweight } \\
(n \text { 820) }\end{array}$ & Significancet & $\begin{array}{l}\text { Obese } \\
(n \text { 820) }\end{array}$ & Significance $†$ \\
\hline Constant & $28 \cdot 25$ & ** & 0.61 & $(*)$ & 0.27 & \\
\hline Family meals & $0 \cdot 10$ & & -0.01 & & 0.00 & \\
\hline Female & $-1 \cdot 54$ & ** & $-1 \cdot 12$ & ** & -0.26 & \\
\hline Age & 0.04 & ** & 0.02 & $\star \star$ & 0.01 & \\
\hline Black, non-Hispanic $\ddagger$ & $2 \cdot 35$ & ** & 0.69 & * & 0.58 & $(*)$ \\
\hline Hispanic & 1.53 & * & 0.48 & & 0.43 & \\
\hline Other/multiple race & 0.03 & & 0.22 & & $-0 \cdot 19$ & \\
\hline Married & -0.38 & & $0 \cdot 12$ & & -0.01 & \\
\hline Any children in household & $1 \cdot 72$ & $(\star)$ & 0.46 & & 0.56 & \\
\hline Years of education & -0.28 & ** & -0.08 & * & $-0 \cdot 15$ & ** \\
\hline Employed full-time, year-round & 0.72 & $(*)$ & 0.25 & & $0 \cdot 19$ & \\
\hline Any children $\times$ family meals & $-0 \cdot 24$ & $(*)$ & -0.06 & & -0.06 & \\
\hline
\end{tabular}

Significance: ${ }^{(*)} P<0 \cdot 10,{ }^{*} P<0 \cdot 05,{ }^{*} P<0.01$.

tThe statistical significance of linear regression coefficients was examined using $t$ tests, and of logistic regression coefficients using Wald statistics. ¥White, non-Hispanic is the reference group.

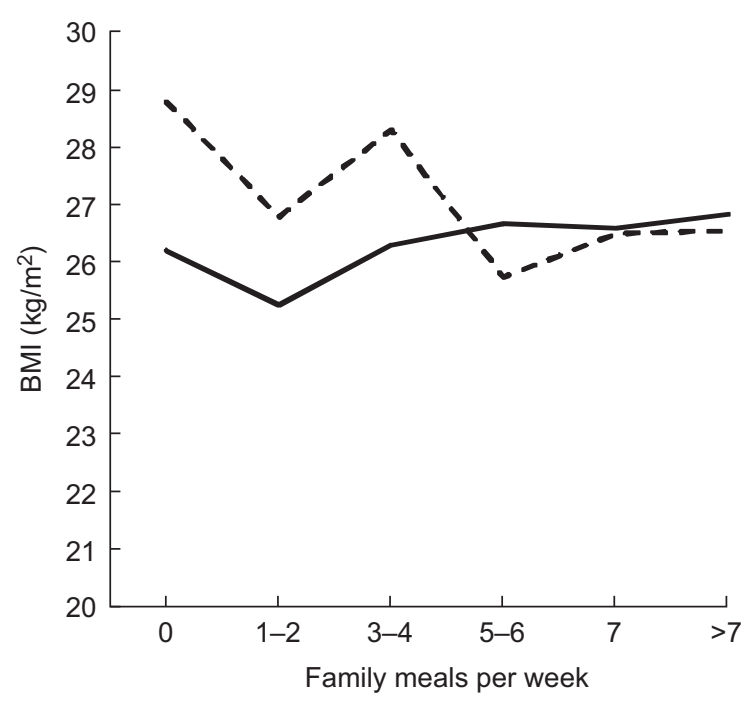

Fig. 1 Regression-adjusted mean BMI by family meals per week for adults in households with $(-\ldots-)$ and without (-) children: nationally representative US adult sample, 2009 Cornell National Social Survey. Regression adjustment included gender, age, race, married, years of education and full-time, year-round employment
A minority of this sample reported that they had no family members to eat with and therefore could not eat family meals. Sociodemographically, these individuals were older and more likely to be unmarried, not have children at home and not be employed full-time yearround. In studying family meals, it is important to recognize that some people do not have family members in their household and therefore cannot eat family meals as defined here; they should be excluded from family meal analyses so that they are not confused with people who live with family members but do not eat with them.

We found that the majority of adults (53\%) reported eating family meals seven or more times per week, and $72 \%$ five or more times per week. These data provide a current national estimate of the frequency of family meals among adults in the USA and are consistent with studies in more limited US samples ${ }^{(13,14)}$.

Our estimates revealed more frequent family meals than have studies of adults in other nations ${ }^{(10-12)}$ and most studies of adolescents ${ }^{(4)}$. Differences in the prevalence of family meals may have occurred because of methodological variations, including differences in samples from 
different places and cultures, variations in sampling procedures, disparities in the questions used to assess family meals, and our exclusion of respondents who did not have family members with whom they could eat from our analytic samples. The higher prevalence of family meals among adults did not appear to occur because adult-only households more frequently ate family meals, because we found that the presence of children in the household was not a significant predictor of the frequency of family meals. Differences between adolescent and adult reporting of family meals may have occurred because adults better remember family meals, have a broader definition of family meals, are more committed to eating family meals than younger people, and place a higher priority on family meals than do adolescents who may skip family meals more often than adults ${ }^{(6)}$.

The findings about the sociodemographic predictors of family meals revealed that being married and not being employed full-time year-round were significantly associated with more frequent family meals in adults. Married individuals may eat more frequent family meals because they share joint households and a partnership where eating together is a marital ideal ${ }^{(32)}$. Individuals who are employed full-time year-round may eat family meals less frequently because of time demands of work and commuting, compared with part-time and seasonal workers, unemployed, retired and disabled individuals who have greater time available to them ${ }^{(40)}$. This finding of an inverse association between adult employment and frequency of family meals is congruent with some studies of adults ${ }^{(12)}$ and adolescents ${ }^{(4)}$. Overall, both marriage and employment provide social structures that may influence patterns of family meals among adults. These two variables do not apply directly to children and most adolescents and therefore were not highlighted in studies of younger individuals, but they do provide insights about social structures that influence family meals.

We found no overall association between frequency of family meals and any of the measures of body weight. This is congruent with a few studies of adolescents that reported no relationship ${ }^{(8,25,27)}$, but incongruent with the majority of adolescent studies that reported an inverse relationship ${ }^{(1-3)}$. However, a significant interaction in our analysis suggests that among adults there may be an inverse association between family meals and BMI in households with children, and no association between family meals and body weight for adults without children. In this model, adults in households with children were marginally heavier overall, but this was concentrated among adults with fewer family meals.

There are three potential explanations for the observed association between family meals and body weight only among adults with children: (i) family meals may influence the body weight of parents; (ii) parents' body weight may influence the frequency of family meals; or (iii) both may be affected by other factors. It is important to consider all of these possible explanations because our cross-sectional data do not allow us to clearly establish the direction of causation.

First, frequency of family meals may influence parental body weight by encouraging parents to engage in social control over their own and their children's eating ${ }^{(41)}$. Eating as a family with children present may promote parental modelling of healthy food intake for children ${ }^{(32)}$ that moderates and minimizes food intake in an effort to promote a healthy meal environment, which influences the weight of the child and the parents. Family meals may also physiologically influence the body weight of parents by slowing their rate of eating ${ }^{(42)}$.

Second, the body weight of parents may influence the frequency of family meals if heavier parents prepare fewer family meals. Sociologically, this might occur if heavier parents do not desire to serve as models of eating behaviour for their children, if they eat foods that they consider inappropriate for children, or if they engage in dieting or overeating behaviours that are incompatible with family meals. Also, if heavier parents are trying to lose weight, they may promote fewer family meals in order to avoid engaging in food shopping and preparation which is associated with additional food intake and higher body weights ${ }^{(43)}$.

Third, frequency of family meals and body weight may both be caused by other psychological, social, economic and cultural factors. For example, complex schedules, conflicting food preferences or family discord may lead to parental stress. Stress, in turn, may lead both to less frequent family meals and weight gain. Similarly, parents who generally conform more loosely to social norms may not participate in the ritual of family eating and also may have less desire to maintain a healthy body weight ${ }^{(44)}$.

While some prior studies had suggested unhealthy impacts of some types of family meals on parents' weights ${ }^{(29)}$, our findings suggest that, overall, family meals are associated with slightly lower body weights for parents. However, the CNSS data did not assess either food settings or food sourcing for family meals. Considering food settings like family meals at home, at the homes of others and at restaurants may help to understand these inconsistent findings. Food sourcing, like whether the food for family meals was cooked at home, prepared in a restaurant or had other origins, may also offer additional insight about family meals and body weight ${ }^{(45)}$.

There are several limitations to the present study which deserve special note. While the sample was sizeable and representative of the USA, it was not sufficiently large to conduct separate stratified analysis of sub-populations like ethnic minorities. Cultural differences in family meals and associations with body weight may be particularly important to understand in the targeting of interventions which promote healthy eating or attempt to facilitate body-weight management. Measurement limitations include potential self-report biases, particularly socially desirable 
responses to questions about family meals and body weight. Under-reporting of weight ${ }^{(36)}$ would be expected to underestimate the relationships observed in the present analysis.

These findings have several implications and applications. They provide novel large-scale survey data about family meals and body weight among a broad cross-section of adults in the USA. Most adults report eating family meals daily, which is more often than adolescents report eating family meals. Marriage is associated with more frequent family meals among adults and full-time year-round employment is related to fewer family meals. Family meals are only marginally associated with body weight among adults living with children and not associated among adults not living with children. Our results are important to consider in counselling, educational, programme and policy decisions about providing and promoting family meals. Future studies about family meals would benefit from broader sampling of adults and young people, larger samples which contain sufficient numbers of respondents from diverse populations and settings to facilitate subgroup analysis, assessment of multiple members of the same households, and consideration of both nuclear and extended family meals. Further, while the present investigation focused on body weight as one potential outcome of family meals, future work should examine other health, psychological and social outcomes ${ }^{(46)}$ which may be associated with family meals.

\section{Acknowledgements}

The research received no specific grant from any funding agency in the public, commercial or not-for-profit sector. The authors declare no conflicts of interest for this study. J.S. and K.H. participated in design of the study and writing the manuscript, and K.H. conducted the data analysis.

\section{References}

1. Sen B (2006) Frequency of family dinner and adolescent body weight status: evidence from the National Longitudinal Survey of Youth, 1997. Obesity (Silver Spring) 14, 2266-2276

2. Gillman MW, Rifas-Shiman SL, Frazier L et al. (2000) Family dinner and diet quality among older children and adolescents. Arch Fam Med 9, 235-240.

3. Taveras EM, Rifas-Shiman SL, Berkey CS et al. (2005) Family dinner and adolescent overweight. Obes Res 13, 900-906.

4. Neumark-Sztainer D, Larson NI, Fulkerson JA et al. (2010) Family meals and adolescents: what have we learned from Project EAT (Eating Among Teens)? Public Health Nutr 13, 1113-1121

5. Boutelle KN, Lytle LA, Murray DM et al. (2001) Perceptions of the family mealtime environment and adolescent mealtime behavior: do adults and adolescents agree? J Nutr Educ 33, 128-133.
6. Fulkerson JA, Neumark-Sztainer D \& Story M (2006) Adolescent and parent views of family meals. J Am Diet Assoc 106, 526-532.

7. Fulkerson JA, Neumark-Sztainer D, Hannan PJ et al. (2008) Family meal frequency and weight status among adolescents: cross-sectional and 5-year longitudinal associations. Obesity (Silver Spring) 16, 2529-2534.

8. Woodruff SJ, Hanning RM, McGoldrick K et al. (2010) Healthy Eating Index-C is positively associated with family dinner frequency among students in grades 6-8 from southern Ontario, Canada. Eur J Clin Nutr 64, 454-460.

9. Anderson SE \& Whitaker RC (2010) Household routines and obesity in US preschool-aged children. Pediatrics $\mathbf{1 2 5}$, 420-428.

10. Warde A \& Martens L (2000) Eating Out: Social Differentiation, Consumption and Pleasure. New York: Cambridge University Press.

11. Pettinger C, Holdsworth M \& Gerber M (2006) Meal patterns and cooking practices in Southern France and Central England. Public Health Nutr 9, 1020-1026.

12. Holme L (2001) Family meals. In: Eating Patterns: A Day in the Lives of Nordic Peoples. Report no. 7-2001, pp. 199-212 [U Kjaernes, editor]. Lysaker: National Institute for Consumer Research.

13. Putnam R (2000) Bowling Alone: The Collapse and Revival of American Community. New York: Simon and Schuster.

14. Herbst KC \& Stanton JL (2007) Changes in family dynamics predict purchase and consumption. $B r$ Food $J$ 109, 648-655.

15. McIntosh WA, Dean W, Torres CC et al. (2009) The American family meal. In Meals in Science and Practice: Interdisciplinary Research and Business Applications, pp. 190-218 [HL Meiselman, editor]. New York: CRC Press.

16. Gable S \& Chang Y \& Krull JL (2007) Television watching and frequency of family meals are predictive of overweight onset and persistence in a sample of school-aged children. J Am Diet Assoc 107, 53-61.

17. Kubrick MY, Davey C, Fulkerson JA et al. (2009) Alternative high school students: prevalence and correlates of overweight. Am J Health Behav 33, 600-609.

18. Yuasa K, Sei M, Takeda E et al. (2008) Effects of lifestyle habits and eating meals together with the family on the prevalence of obesity among school children in Tokushima, Japan: a cross-sectional questionnaire-based survey. $J$ Med Invest 55, 71-77.

19. Gundersen C, Lohman B, Eisenmann JC et al. (2008) Childspecific food insecurity and overweight are not associated in a sample of 10- to 15-year-old low-income youth. J Nutr 138, 371-378.

20. Mikkila V, Lahti-Koski M, Pietinen P et al. (2003) Associates of obesity and weight dissatisfaction among Finnish adolescents. Public Health Nutr 6, 49-56.

21. Rollins BY, Belue RZ \& Francis LA (2010) The beneficial effect of family meals on obesity differs by race, sex, and household education: the National Survey of Children's Health, 2003-2004. J Am Diet Assoc 110, 1335-1339.

22. BeLue R, Francis LA, Rollins B et al. (2009) One size does not fit all: identifying risk profiles for overweight in adolescent population subsets. J Adolesc Health 45 , $517-524$

23. Utter J, Scragg R, Schaaf D et al. (2008) Relationships between frequency of family meals, BMI and nutritional aspects of home food environment among New Zealand adolescents. Int J Behav Nutr Phys Act 5, 50.

24. Gable S \& Lutz S (2000) Household, parent, and child contributions to childhood obesity. Fam Relat 49, 293-300.

25. Mamun AA, Lawlor DA, O'Callaghan MJ et al. (2005) Positive maternal attitude to the family eating together decreases the risk of adolescent overweight. Obes Res 13, 1422-1430. 
26. Woodruff SJ \& Hanning RM (2009) Associations between family dinner frequency and specific food behaviors among grade six, seven, and eight students from Ontario and Nova Scotia. J Adolesc Health 44, 431-436.

27. Wurback A, Zellner K \& Kromeyer-Hauschild K (2009) Meal patterns among children and adolescents and their associations with weight status and parental characteristics. Public Health Nutr 12, 1115-1121.

28. Price JL, Day RD \& Yorgason JB (2009) A longitudinal examination of family processes, demographic variables, and adolescent weight. Marriage Fam Rev 45, 310-330.

29. Boutelle KN, Fulkerson JA, Neumark-Sztainer D et al. (2007) Fast food for family meals: relationships with parent and food intake, home food availability and weight status. Public Health Nutr 10, 16-23.

30. Hobbs F (2005) Examining American Household Composition: 1990 and 2000. US Census Bureau Census 2000 Special Reports no. CENSR-24. Washington, DC: US Government Printing Office.

31. Cherlin A (2004) The deinstitutionalization of marriage. J Marriage Fam 66, 848-861.

32. Bove CF, Sobal J \& Rauschenbach BS (2003) Food choices among newly married couples: convergence, conflict, individualism, and projects. Appetite 40, 25-41.

33. McIntosh WA (1999) The family meal and its significance in global times. In Food in Global History, pp. 217-239 [R Grew, editor]. Boulder, CO: Westview Press.

34. Capagrossi A \& Miller Y (2010) Cornell National Social Survey 2009, Report 1: Introduction \& Methodology. Ithaca, NY: Cornell University Survey Research Institute.

35. World Health Organization (1995) Physical Status: The Use and Interpretation of Anthropometry. Report of a WHO Expert Committee. Technical Report Series no. 854. Geneva: WHO.
36. Gorber SC, Tremblay M, Moher D et al. (2007) A comparison of direct vs. self-reported measures for assessing height, weight and body mass index: a systematic review. Obes Rev 8, 307-326.

37. Sobal J, Hanson KL \& Frongillo EA (2009) Gender, ethnicity, marital status, and body weight in the United States. Obesity (Silver Spring) 17, 2223-2231.

38. Flegal KM, Carroll MD, Ogden CL et al. (2010) Prevalence and trends in obesity among US adults, 1999-2008. JAMA 303, 235-241.

39. Jaccard J \& Turrisi R (2003) Interaction Effects in Multiple Regression, 2nd ed. Thousand Oaks, CA: Sage.

40. Jabs J \& Devine CM (2006) Time scarcity and food choices: an overview. Appetite 47, 196-204.

41. Umberson D (1992) Gender, marital status and the social control of health behavior. Soc Sci Med 34, 907-917.

42. Kokkinos A, Roux CW, Alexiadou K et al. (2010) Eating slowly increases the postprandial response of the anorexigenic gut hormones, peptide YY and glucagon-like peptide-1. J Clin Endocrinol Metab 95, 333-337.

43. Larson NI, Story M, Eisenberg ME et al. (2006) Food preparation and purchasing roles among adolescents: associations with sociodemographic characteristics and diet quality. J Am Diet Assoc 106, 211-218.

44. Block JP, Zaslavsky AM, Ding L et al. (2009) Psychosocial stress and change in weight among US adults. $A m J$ Epidemiol 170, 181-192.

45. McIntosh WA, Kubena KS, Tolle G et al. (2010) Mothers and meals. The effects of mothers' meal planning and shopping motivations on children's participation in family meals. Appetite 55, 623-628.

46. Story M \& Neumark-Sztainer D (2005) A perspective on family meals. Do they matter? Nutr Today 40, 261-266. 